\title{
Microstresses in Thermally Stable Diamond Composites made by High Pressure Infiltration Technique
}

\author{
V. Luzin ${ }^{1, a^{*}}$, G. Voronin ${ }^{2, b}$, M. Avdeev ${ }^{1, c}$ and J. Boland ${ }^{3, d}$ \\ ${ }^{1}$ Australian Nuclear Science and Technology Organisation, Lucas Heights, NSW, 2232 Australia \\ ${ }^{2}$ Smith MegaDiamond Inc, Schlumberger Co, Provo, UT 84604, USA \\ ${ }^{3}$ CSIRO Earth Science and Resource Engineering, Pullenvale, QLD 4069, Australia \\ avladimir.luzin@ansto.gov.au, bgvoronin@hotmail.com, 'cmax.avdeevn@ansto.gov.au, \\ dJim.Boland@csiro.au
}

Keywords: Residual Stress, Diamond Composite, Microstress

\begin{abstract}
Microstresses in the diamond and $\mathrm{SiC}$ phases of the TSDCs (thermally stable diamond composites), produced by the high pressure infiltration technique, were measured using the neutron diffractometer, KOWARI, at the OPAL research reactor. Microstresses are developed as a result of the cooling and pressure reduction from the sintering high temperature and high pressure (HTHP) conditions. Their magnitude is determined by the thermo-mechanical properties of the $\mathrm{SiC}$ matrix and diamond grit, pressure and temperature conditions as well as the exact TSDC phase composition. The experimental results were interpreted in terms the "matrixinclusion" composite model that was used to evaluate the composite structural integrity.
\end{abstract}

\section{Introduction}

Diamonds composites are finding increasing use in mining, manufacturing and civil construction industries as cutting elements and drilling bits of various toolings. Although diamond is extremely brittle with low impact strength, the diamond composites have superior hardness and toughness better suited to these applications.

There are two diamond-based composite systems. So-called PCD (polycrystalline diamond composite) has a certain amount of metal binder phase, usually Co. The use of the metal binder restricts the operational temperature range for PCD to less than $800^{\circ} \mathrm{C}$. The second system of diamond composites is TSDC, which extends the temperature range up to $1400^{\circ} \mathrm{C}$ [1]. TSDC is free of any metal binder, but a ceramic binder is used instead, usually SiC.

There are several methods of producing TSDC, but most commonly the diamond-SiC composites are produced by the liquid Si infiltration techniques involving reactive sintering. They share some common features to form $\mathrm{SiC}$ matrix/binder for the diamond particles since they are all based on reactive bonding operations, usually in HPHT conditions. During this operation, molten silicon infiltrates the diamond powder, reacts with the diamond powders that results in the formation of the diamond-SiC composite. On the other hand, the HPHT conditions can vary drastically. In some methods the sintering is carried out in the diamond stability region $\left(\sim 1500^{\circ} \mathrm{C}\right.$ and $\left.\sim 5.5 \mathrm{GPa}\right)$, while others operate in the graphite stable region $\left(\sim 1500^{\circ} \mathrm{C}\right.$ and $\sim 2.0-$ $3.5 \mathrm{GPa}$ ) as in case of the process patented by Ringwood in 1980s [2]. There are some variations of the method that involve no pressure or very low pressure $[3,4]$. In some cases pressure can be as high as $8-10 \mathrm{GPa}$ and temperature as high as $1800-2000^{\circ} \mathrm{C}$ [5]. While the first two cases were analyzed for stress earlier [6], the later production route, high pressure silicon infiltration technique [5], is the focus of the current investigation.

While thermal stability is a very important property for a tool's performance, other properties such as impact resistance, thermal fatigue, fracture toughness and wear resistance are related to 
the stress state of the material. The residual stress originates from the manufacturing process, which is usually HPHT process, while the applied stress comes from severe mechanical/thermal loading these composites are subjected to during operation.

Since the current TSDC manufacturing processes involve such high temperatures and pressures, the residual stresses in such composite materials arise from (i) thermal mismatch, due to the difference in thermal expansion coefficient between the diamond and $\mathrm{SiC}$ matrix phase during the quenching to room temperate and (ii) elastic mismatch, due to the difference in bulk elastic modulus between diamond and $\mathrm{SiC}$ matrix phases during the pressure drop from high to ambient.

The infiltration process of liquid silicon into the diamond green body is a complex phenomenon. It involves percolation of liquid $\mathrm{Si}$, dissolution of carbon into $\mathrm{Si}$, reaction and formation of $\mathrm{SiC}$. This process is particle-size sensitive and in certain conditions can lead to closure of pores in the diamond compact due to the sealing effect of the $\mathrm{SiC}$ formation. This effect is more pronounced for smaller particle sizes of the diamond green body and is also affected by the pressure conditions of the process when diamond particles experience fracturing with effective decrease in the particle size. All these processing variables, including even sample shape and size, can eventually lead to formation of specific microstructures (mostly micro-defect structures such as micro-porosity, micro-cracking and residual silicon) as well as residual microstresses.

\section{Samples}

In the current study the focus is on the high pressure (8-10 GPa) high temperature (1800$2000^{\circ} \mathrm{C}$ ) variation of the sintering technology [5]. Samples of two geometries were produced under the same high temperature $\left(\sim 1900^{\circ} \mathrm{C}\right)$ and high pressure $(\sim 9 \mathrm{GPa})$ conditions. The two sample geometries were (i) cylinders with diameter of $3 \mathrm{~mm}$ and height of $7 \mathrm{~mm}$ and (ii) $6 \mathrm{~mm}$ side triangular prisms with height of $4 \mathrm{~mm}$ (Fig. 1). For the cylindrical samples, finer diamond powder was used in conjunction with coarser grain size $(30-40 \mu \mathrm{m})$ to provide better infiltration for the whole sample height. For the triangular prism samples finer diamond grit $(20-30 \mu \mathrm{m})$ was used (Fig. 2). With two different diamond particle sizes and two shapes, the percolation conditions might differ, forming, as discussed above, somewhat different micro-mechanical systems characterized also by different stresses.

Two representative samples for each of the geometries were studied. Samples were characterized in the "as-manufactured" condition with no service history.

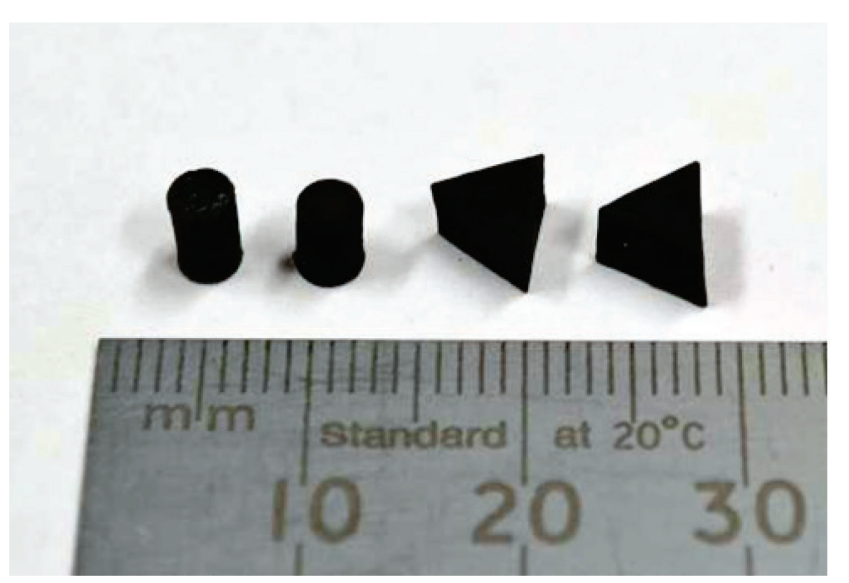

Fig. 1: Samples geometries.

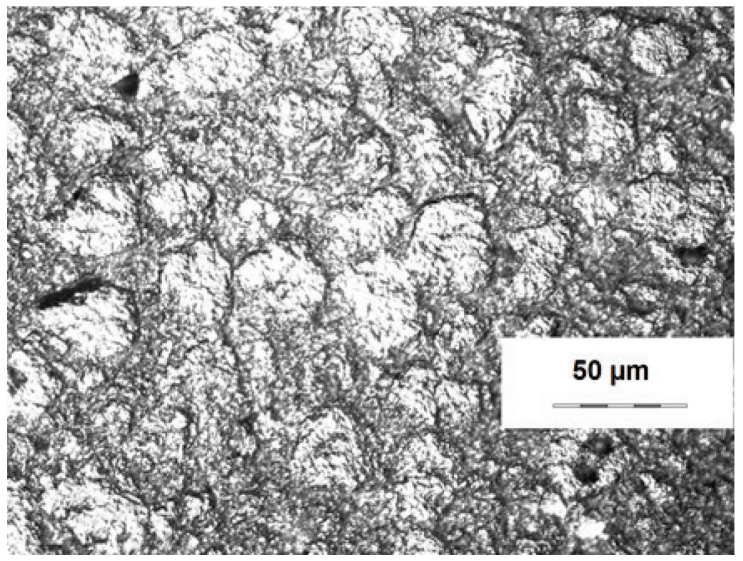

Fig. 2: Microstructure: optical microscopy image of the triangular sample surface. 


\section{Experimental: phase and stress analyses}

Two characterizations were conducted by means of neutron diffraction using diffractometers of the Australian research reactor OPAL.

Phase identification and quantitative phase analysis were done at the neutron high-resolution powder diffractometer, WOMBAT [7]. Neutron diffraction patterns were collected in the range of $25^{\circ}$ to $145^{\circ}$ using wavelength of $1.54 \AA$ and WOMBAT's $120^{\circ}$-range large position sensitive detector. The patterns were analysed using Rietveld refinement technique (GSAS Rietveld refinement software with the EXPGUI interface [8]) to extract volume fractions of diamond and $\beta$-SiC (a cubic polymorph, formed at sintering temperatures below $1700^{\circ} \mathrm{C}$ ). No other polymorphs of $\mathrm{SiC}$ or $\mathrm{C}$ and no residual $\mathrm{Si}$ were detected. The accuracy of phase fraction evaluation was better than $0.2 \%$.

Residual stress analysis was carried out using the neutron residual stress diffractometer, KOWARI [9]. In the residual stress experiment both phases of TSDC samples were measured: diamond (311) reflection and $\mathrm{SiC}(220)$ reflection at a wavelength of $1.52 \AA$. Bragg angles of these reflections were $90^{\circ}$ and $58.4^{\circ}$ respectively. Due to the overall size of the samples, a relatively small gauge volume with dimension $1.5 \times 1.5 \times 4 \mathrm{~mm}^{3}$ was used so that measurements could be made from the bulk of the smallest sample. During measurement the gauge volume was positioned in the center of each sample and the sample was rocked in the range of $20^{\circ}$ to improve the grain statistics. The measurement time was 10-15 min for the diamond phase and 2-3 hours for the SiC phase.

A special test was made to check isotropy and uniformity of one fine grained (triangular prism) sample by measuring it in multiple directions and several different locations. Since no statistically significant variation in the lattice parameter was found, isotropy and uniformity was confirmed in this way and assumed in the following data analysis.

Determination of the unstressed $\mathrm{d}$-spacing, $\mathrm{d}_{0}$, is critical for correct microstrain and stress determination in composite materials; therefore it received special attention. The $\mathrm{d}_{0}$ values for both phases, $d_{0}^{S i C}$ and $d_{0}^{\text {Dia }}$, were assumed to be the same in all four samples considering the same treatment history. In principle, it is sufficient to use two equations, corresponding to two samples, setting macrostress to zero, $\sigma_{i}^{\text {Macro }}=f_{i}^{S i C} \sigma_{i}^{S i C}+$ $f_{i}^{\text {Dia }} \sigma_{i}^{\text {Dia }}=0, i=1,2$ in order to resolve the system of two equations with two unknowns, $d_{0}^{S i C}$ and $d_{0}^{\text {Dia }}$, and determine them in a unique way (assuming that $f_{1} \neq f_{2}$ to avoid degeneracy). In the above equation $f_{i}^{\alpha}$ stands for the volume fraction of the $\alpha$-th phase and $\sigma_{i}^{\alpha}$ is the total hydrostatic phase stress, $\sigma_{i}^{\alpha}=\frac{1}{1 / 2 S_{2}^{\alpha}(h k l)+3 S_{1}^{\alpha}(h k l)} \frac{d_{i}^{\alpha}-d_{o}^{\alpha}}{d_{0}^{\alpha}}, \alpha=$ SiC , Dia. In the case of four samples, the system of such equations is overdetermined, therefore, $\mathrm{d}_{0}$ values were defined in such a way that provided the condition of zero macrostress in all four samples simultaneously in the leastsquares minimization sense (minimizing the

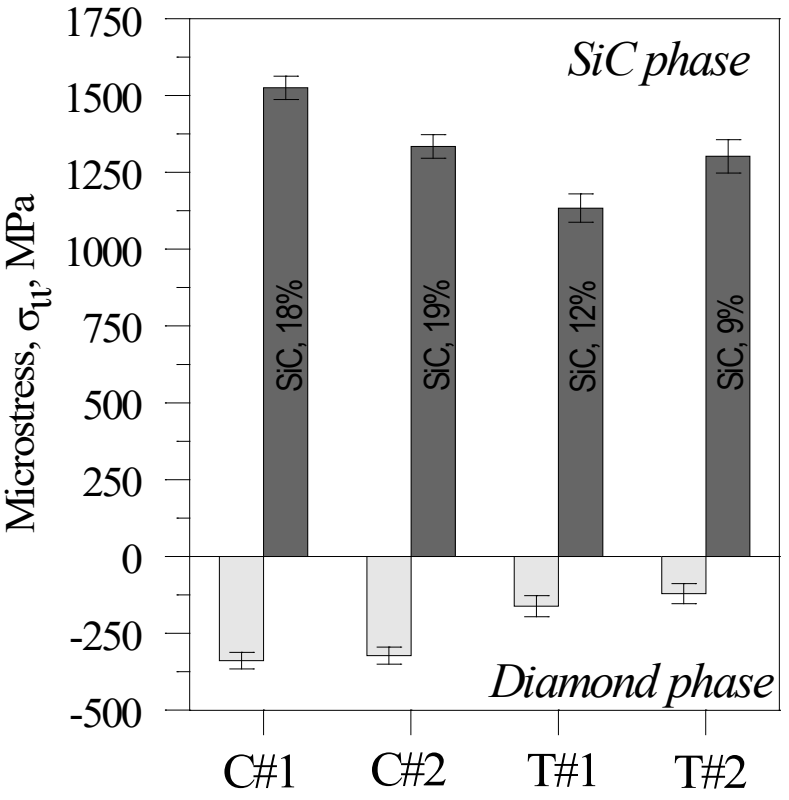

Fig. 3: Microstress of the $\mathrm{SiC}$ and diamond phases in the cylindrical $(C)$ and triangular (T) samples. 
sum of all four squared macrostress residuals). Treated this way, the $\mathrm{d}_{0}$ for diamond and $\mathrm{SiC}$ were determined with accuracy of the same order of magnitude as the accuracy of the measurements, $\sim 1 \times 10^{-5}$ for diamond and $\sim 5 \times 10^{-5}$ for SiC.

Strain-to-stress calculations were carried out using appropriate hkl-dependent diffraction elastic constants calculated from the single crystal elastic constants of diamond and $\mathrm{SiC}$ [10]. Numerically, $\mathrm{S}_{1}(\mathrm{hkl})$ and $1 / 2 \mathrm{~S}_{2}(\mathrm{hkl})$ were -0.28 and $1.42 \mathrm{TPa}^{-1}$ for the diamond (311) reflection, while for the $\mathrm{SiC}$ (220) reflection they were -0.40 and $2.71 \mathrm{TPa}^{-1}$.

\section{Results and discussion}

The results of the neutron phase analysis and neutron stress analysis, based on experimentally determined phase composition are shown in Table 1 and visually illustrated in Fig. 3 . With the accuracy of the phase analysis being better than 0.2 vol.\%, the uncertainty in the volume fraction determination does not make any significant contribution to the overall uncertainty of the stress values. The finite strain measurement accuracy, $\sim 15 \mu$ strain for diamond and 30-50 $\mu$ strain for $\mathrm{SiC}$ due to the neutron counting statistics is the major source of uncertainties of stress that are reported in Table 1. Additionally, some inaccuracy in $\mathrm{d}_{0}$ 's and/or the single crystal elastic constants can result in somewhat bigger errors.

The experimental check for isotropy not only suggests isotropy of the microstructure and its properties but was used as an assumption in the stress analysis so that one stress component, the hydrostatic microstress (or pressure), fully characterizes the stress state.

Table 1. Experimentally determined phase composition and phase microstresses.

\begin{tabular}{|l|c|l|l|l|}
\hline Sample ID & $\begin{array}{l}\text { Weight fraction } \\
\text { (diamond/SiC) }\end{array}$ & $\begin{array}{l}\text { Volume fraction } \\
\text { (diamond/SiC) }\end{array}$ & $\begin{array}{l}\text { Stress in } \\
\text { diamond [MPa }]\end{array}$ & $\begin{array}{l}\text { Stress in SiC } \\
{[\mathrm{MPa}]}\end{array}$ \\
\hline Cylindrical samples \\
\hline C\#1 & $0.83 / 0.17$ & $0.82 / 0.18$ & $-339 \pm 27$ & $1524 \pm 40$ \\
\hline C\#2 & $0.82 / 0.18$ & $0.81 / 0.19$ & $-323 \pm 28$ & $1334 \pm 40$ \\
\hline \multicolumn{5}{|l|}{ Prism samples } \\
\hline T\#1 & $0.88 / 0.12$ & $0.88 / 0.12$ & $-162 \pm 35$ & $1133 \pm 46$ \\
\hline T\#2 & $0.92 / 0.08$ & $0.91 / 0.09$ & $-121 \pm 33$ & $1302 \pm 54$ \\
\hline
\end{tabular}

\section{Discussion}

The results of the stress analysis on the samples made by the high pressure infiltration technique are consistent with other TSDCs [6], with hydrostatic microstresses in diamond phase reaching several hundreds of $\mathrm{MPa}$ in compression equilibrated by tensile microstresses in $\mathrm{SiC}$ with magnitudes up to GPa and higher.

Interpretation of the results can be made using a micromechanical model of the generalized particulate composite employing spherical inclusion theory. The stress analysis by means of the Hashin-Shtrikman bounds [11], rather than relying on one specific model, assumes isotropic and particulate microstructure and provides a range of possibilities for matrix-inclusion interaction. In this approach the hydrostatic microstress can be evaluated as a function of a temperature or external pressure drop. The calculations based on a temperature change from the sintering to room temperature are given Fig. 4 in comparison with the experimental data. It is evident that all samples generally match the lower HS bound ("HS-", diamond matrix, SiC inclusions) very well. This suggests an inter-connectivity of the diamond particles so the diamond phase topology can be considered as a continuous framework with islands of SiC. 
With such good agreement between experimental and calculated stresses, some implications can be deduced.

(1) There is no pressure-drop-generated component in the overall stress state. If there was such a contribution present with pressure drop of $\Delta \mathrm{P}=9 \mathrm{GPa}$, then the overall expected stresses would be significantly lower, e.g some $-40 \mathrm{MPa}$ rather than the -340 $\mathrm{MPa}$ experimentally determined for the diamond phase in the cylindrical samples. Thus only thermally generated stresses appear through the production pathway when the external pressure is released immediately, while temperature is reduced to ambient temperature at a slower rate. The same conclusion was made through surface stress analysis by means of Raman spectroscopy of the TSDC samples produced by the same technique [12] - the magnitude of residual stress is primarily dependent on temperature conditions, not pressure change.

(2) In the same way it can be deduced that there is no (or at least no significant) microcracking present in the samples, otherwise a significant stress relaxation with significantly lower absolute values of the microstresses would be detected. However, some speculations can be made regarding the triangular-shaped samples, where some stress relaxation can be observed, approximately $80 \%$ from the expected values. The fact that the grain size of the diamond powder was smaller for these samples can be reflected in higher probability of the sealing effect with formation of isolated micro-pores. This can be responsible for the elastic weakening of the composite and can contribute to some stress relaxation.

\section{Conclusions}

Two types of TSDC samples (diamond-SiC composite), produced by the silicon infiltration technique at HTHP conditions, were analyzed by means of neutron diffraction. Phase composition was obtained through the full diffraction pattern analysis to assist in the residual stress analysis. Neutron diffraction strain analysis was done in a single peak experiment and microstresses (phase incompatibility) were determined in the two phases, compressive in the diamond phase and tensile in the $\mathrm{SiC}$ phase.

The thermal nature of the experimentally determined microstress was deduced through the micromechanical analysis of the composite. The results suggest that almost all thermally generated microstresses are preserved in the samples. No significant sign of microcracking and stress relaxation was found, thus confirming the micromechanical integrity of the samples and suggesting good quality and performance of the TSDC samples. 


\section{References}

[1] J.N. Boland and X.S. Li, Microstructural characterisation and wear behaviour of diamond composite materials, Materials, 3 (2010) 1390-1419. https://doi.org/10.3390/ma3021390

[2] A.E. Ringwood, Diamond compacts and process for making same. Patent 4948388, (1989).

[3] K. Mlungwane, M. Herrmann and I. Sigalas, The low-pressure infiltration of diamond by silicon to form diamond-silicon carbide composites, J. Eur. Ceram. Soc. 28 (2008) 321-326. https://doi.org/10.1016/j.jeurceramsoc.2007.06.010

[4] C. Zhu, J. Lang and N. Ma, Preparation of Si-diamond-SiC composites by in-situ reactive sintering and their thermal properties, Ceram. Int. 38 (2012) 6131-6136. https://doi.org/10.1016/j.ceramint.2012.04.062

[5] G. Voronin, T. Zerda, J. Gubicza, T. Ungár and S. Dub, Properties of nanostructured diamond-silicon carbide composites sintered by high pressure infiltration technique, J. Mater. Res. 19 (2004) 2703-2707. https://doi.org/10.1557/JMR.2004.0345

[6] V. Luzin, J. Boland, M. Avdeev and X. Li, Characterization of Thermally Stable Diamond Composite Material, Mater. Sci. Forum $777 \quad$ (2014) 165-170. https://doi.org/10.4028/www.scientific.net/MSF.777.165

[7] A.J. Studer, M.E. Hagen and T.J. Noakes, Wombat: The high-intensity powder diffractometer at the OPAL reactor, Physica B: Condensed Matter 385 (2006) 1013-1015. https://doi.org/10.1016/j.physb.2006.05.323

[8] B. Toby, EXPGUI, a graphical user interface for GSAS, J. Appl. Crys. 34 (2001) 210-213. https://doi.org/10.1107/S0021889801002242

[9] O. Kirstein, V. Luzin and U. Garbe, The Strain-Scanning Diffractometer Kowari, Neutron News 20 (2009) 34-36.

[10] K.S. Alexandrov and T.V. Ryzhova, The elastic properties of crystals, Sov. Phys. Crys., 6 (1961) 228-252.

[11] Z. Hashin, The Elastic Moduli of Heterogeneous Materials, J. Appl. Mech. 29 (1962) 143150.

[12] M. Wieligor and T. Zerda, Surface stress distribution in diamond crystals in diamondsilicon carbide composites, Diamond Relat. Mater. $17 \quad$ (2008) 84-89. https://doi.org/10.1016/j.diamond.2007.10.035 